\title{
A Note on Weak Keys of PES, IDEA and some Extended Variants
}

\author{
Jorge Nakahara Jr, Bart Preneel, Joos Vandewalle \\ Katholieke Universiteit Leuven, Dept. ESAT/SCD-COSIC, Belgium \\ \{jorge.nakahara,bart.preneel,joos.vandewalle\}@esat.kuleuven.ac.be
}

\begin{abstract}
This paper presents an analysis of the PES cipher in a similar setting as done by Daemen et al. at Crypto'93 for IDEA. The following results were obtained for 8.5-round PES: a linear weak-key class of size $2^{48}$, two differential weak-key classes of size $2^{41}$, and two differentiallinear weak-key classes of size $2^{62}$. For 17 -round PES (double PES): a linear weak-key class of size $2^{7}$, and a differential weak-key class of size $2^{7}$ were found. These attacks demonstrate that doubling the number of rounds in PES is not enough to avoid weak keys. These findings were possible because the cipher structure from PES to IDEA was changed but the key schedule algorithm remained the same. Daemen suggested a modified key schedule for IDEA in order to avoid weak keys. We found a differential weak-key class of size $2^{83}$ for 2.5-round IDEA under his redesigned key schedule, and a differential-linear weak-key class of size $2^{68}$ for 3.5-round IDEA. The presence of weak keys has some consequences. Recall that without weak-key assumptions there are no known attacks on more than 4.5 rounds of IDEA. Furthermore, the existence of weak keys may imply that the block cipher becomes unsuitable in stream cipher and hash function constructions.
\end{abstract}

Keywords: IDEA and PES ciphers, differential, linear and differential-linear weak-keys, cryptanalysis.

\section{Introduction}

The Proposed Encryption Standard (PES) is an iterated block cipher designed by Lai and Massey in 1990 [6]. PES processes 64-bit blocks, under a 128-bit key, and iterates 8 rounds plus an output transformation (OT), Fig. 1. Similarly, the International Data Encryption Algorithm (IDEA) is a 64-bit block cipher, using a 128-bit key, and iterating 8 rounds plus an output transformation, designed by Lai, Massey and Murphy in 1991 [7] (Fig. 2). IDEA was a former candidate block cipher to the NESSIE Project $[8,10]$. The block ciphers IDEA and PES use three group operations: addition in $\mathbb{Z}_{2^{16}}$, denoted by $\boxplus$, bitwise exclusive-or,

\footnotetext{
* This research is supported by the Katholieke Universiteit Leuven, and partially by
} GOA project Mefisto 2000/06 of the Flemish Government.

Appeared in Proceedings of the 6th Information Security Conference (ISC 2003), Lecture Notes in Computer Science 2851, C. Boyd, and W. Mao (eds.), Springer-Verlag, pp. 267-279, 2003. (C)2003 Springer-Verlag 
denoted $\oplus$, and multiplication in $\operatorname{GF}\left(2^{16}+1\right)$, denoted $\odot$, with 0 represented by $2^{16}$.

The key schedule of IDEA and PES is the same and processes the initial 128-bit user key into fifty-two 16-bit subkeys. Each round uses six subkeys, and the output transformation (OT) uses four subkeys. Initially, the 128-bit key is partitioned into eight 16-bit words, which are used as the first eight subkeys. Additional subkeys are generated by successively rotating 25 bits left the 128-bit register consisting of the previous eight subkeys, and partitioning the resulting register into eight 16-bit words, which represent the next eight subkeys. Table 1 shows the dependency of subkey bits on the user key bits, which is indexed from 0 (MSB: most significant bit) to 127 (LSB: least significant bit). Bit 0 is assumed to be positioned to the right of bit 127 , that is, in a circular fashion, due to the rotation operation.

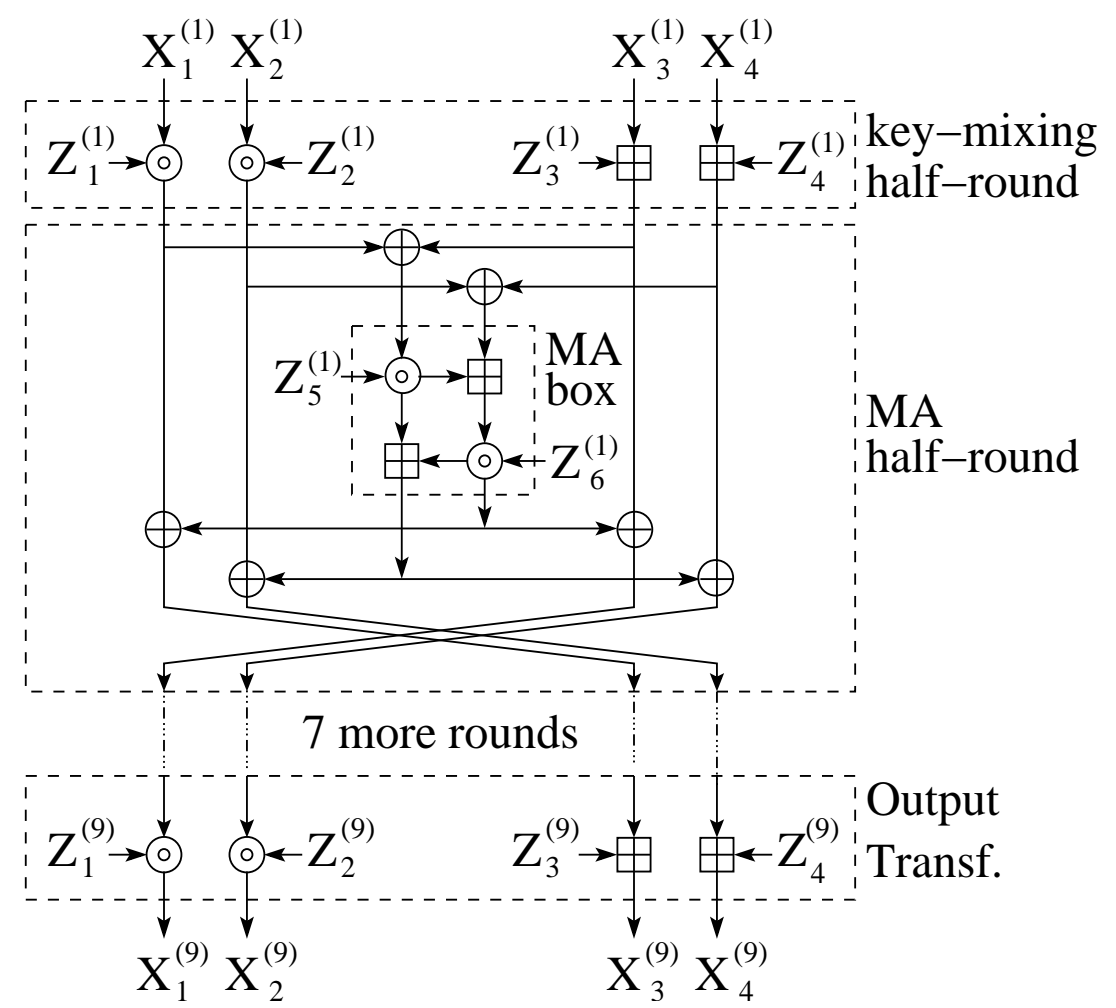

Fig. 1. Computational graph of the PES block cipher

The PES and IDEA ciphers, although defined with 8.5 rounds, can both be extended to a larger number of rounds by just allowing the key schedule to generate further 16-bit subkey words. Attacks will be presented on variable-round

Appeared in Proceedings of the 6th Information Security Conference (ISC 2003),

Lecture Notes in Computer Science 2851, C. Boyd, and W. Mao (eds.), Springer-Verlag, pp. 267-279, 2003. (C)2003 Springer-Verlag 
Table 1. Dependency of subkey bits on the user key bits of IDEA and PES

\begin{tabular}{|c|cccccc|}
\hline$r$-th round & $Z_{1}^{(r)}$ & $Z_{2}^{(r)}$ & $Z_{3}^{(r)}$ & $Z_{4}^{(r)}$ & $Z_{5}^{(r)}$ & $Z_{6}^{(r)}$ \\
\hline 1 & $0-15$ & $16-31$ & $32-47$ & $48-63$ & $64-79$ & $80-95$ \\
2 & $96-111$ & $112-127$ & $25-40$ & $41-56$ & $57-72$ & $73-88$ \\
3 & $89-104$ & $105-120$ & $121-8$ & $9-24$ & $50-65$ & $66-81$ \\
4 & $82-97$ & $98-113$ & $114-1$ & $2-17$ & $18-33$ & $34-49$ \\
5 & $75-90$ & $91-106$ & $107-122$ & $123-10$ & $11-26$ & $27-42$ \\
6 & $43-58$ & $59-74$ & $100-115$ & $116-3$ & $4-19$ & $20-35$ \\
7 & $36-51$ & $52-67$ & $68-83$ & $84-99$ & $125-12$ & $13-28$ \\
8 & $29-44$ & $45-60$ & $61-76$ & $77-92$ & $93-108$ & $109-124$ \\
OT & $22-37$ & $38-53$ & $54-69$ & $70-85$ & - & - \\
\hline
\end{tabular}

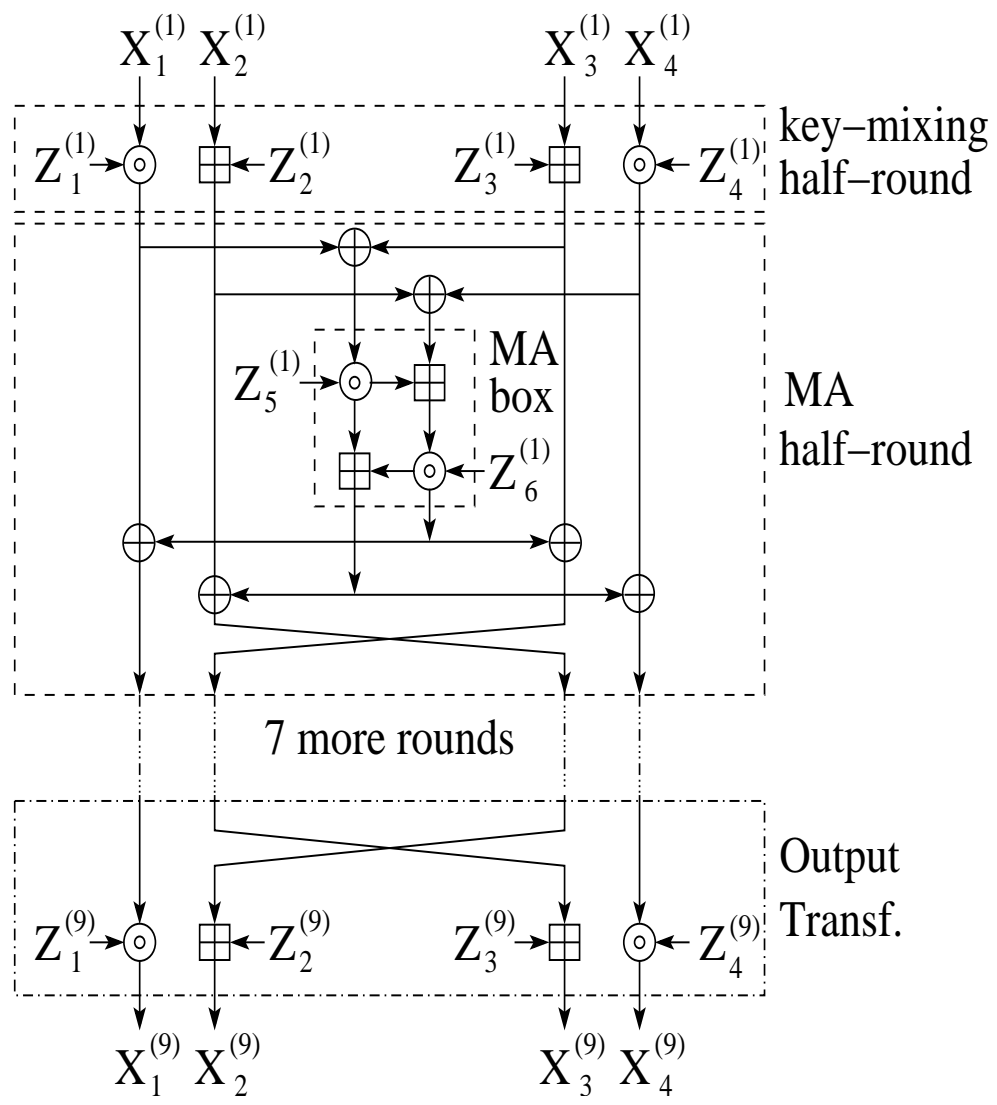

Fig. 2. Computational graph of the IDEA block cipher

Appeared in Proceedings of the 6th Information Security Conference (ISC 2003), Lecture Notes in Computer Science 2851, C. Boyd, and W. Mao (eds.), Springer-Verlag, pp. 267-279, 2003. (C)2003 Springer-Verlag 
variants of PES and IDEA, denoted $r$-round PES and $r$-round IDEA, where $r$ denotes the number of full rounds. Versions including the output transformation will be denoted with the prefix $r .5$-round.

This paper is organized as follows: Sect. 2 presents linear and differential weak-key classes for PES and extended variants. Sect. 3 describes differentiallinear weak-key classes of PES and attacks on reduced-round IDEA with Daemen's modified key schedule. Sect. 4 summarizes the results of the paper.

\section{Linear and Differential Weak Keys}

In [3], Daemen et al. described a class of $2^{23}$ keys for IDEA, that exhibited a linear relation with probability one. These linear relations assume that the multiplicative subkey words $Z_{i}^{(r)}, i \in\{1,4,5,6\}$ have value 0 or 1 . These restrictions are called the weak-key assumptions. Table 2 contains a summary of one-round linear relations and one-round characteristics for PES, under some weak-key assumptions. We follow the same notation as in [3]: $\alpha^{(r)} \stackrel{1 r}{\rightarrow} \alpha^{(r+1)}$ denotes that the $r$-th round 64-bit input mask $\alpha^{(r)}$ causes the 64-bit output mask $\alpha^{(r+1)}$ after one round with bias $\frac{1}{2}$, under some subkey restrictions. Similarly, $\delta_{r} \stackrel{1 r}{\rightarrow} \delta_{r+1}$ denotes that the $r$-th round 64-bit input exclusive-or difference $\delta_{r}$ causes the 64-bit output exclusive-or difference $\delta_{r+1}$ after one round, with probability one, under some restricted subkey words. In Table $2, \nu=2^{15}=8000_{x}$, is a 16-bit difference. Each bit mask or input difference consists of a 4 -tuple for the four 16-bit words in a block.

Based on Table 2, the largest weak-key class for PES uses the one-round iterative linear relation $(0,0,0,1) \stackrel{1 r}{\rightarrow}(0,0,0,1)$, which holds with probability one, under the given subkey restrictions. For the full 8.5-round PES, the weak-key assumption requires that the user key bits numbered 13-48, 66-94, and 109-123 be zero (see Table 1 ). This leaves the user key bits $0-12,49-65,95-108$, and 124-127 unrestricted, resulting in a weak-key class of size $2^{48}$. The bit masks for each intermediate round of PES are shown in Table 3, where $\operatorname{msb}_{n}(Z)$ indicates the $n$ most significant bits of the subkey $Z$ that are zero.

Notice that the linear weak-key class in Table 3 could be extended beyond 8.5 rounds. For example the one-round iterative linear relation $(0,0,0,1) \stackrel{1 r}{\rightarrow}$ $(0,0,0,1)$ concatenated with itself for 16-round PES holds for a weak-key class of size $2^{10}$; for 17 -round PES this relation holds only for the all-zero key. The one-round iterative linear relation $(0,1,0,1) \stackrel{1 r}{\rightarrow}(0,1,0,1)$ holds for 17 -round PES for a weak-key class of size $2^{7}$; for 17.5 -round PES, this linear relation only holds for the all-zero key (this all-zero key is the only key for which there is a linear relation holding for $r$-round PES, for arbitrarily large $r$ ). As for IDEA, the linear relation based on the 3 -round iterative relation $(1,0,0,1) \stackrel{1 r}{\rightarrow}(0,1,0,1) \stackrel{1 r}{\rightarrow}$ $(0,0,1,1) \stackrel{1 r}{\rightarrow}(1,0,0,1)$ extended to 14 rounds, holds for a weak-key class of size $2^{4}$.

Concerning differential characteristics, and referring to the middle column in Table 2, the largest differential weak-key class of PES uses the one-round

Appeared in Proceedings of the 6th Information Security Conference (ISC 2003),

Lecture Notes in Computer Science 2851, C. Boyd, and W. Mao (eds.), Springer-Verlag, pp. 267-279, 2003. (C)2003 Springer-Verlag 
Table 2. One-round linear relations and characteristics for PES

\begin{tabular}{|c|c|c|c|c|c|}
\hline & & \multicolumn{4}{|c|}{ Conditions on Subkeys } \\
\hline$\alpha^{(r)} \stackrel{1 r}{\longrightarrow} \alpha^{(r+1)}$ & $\delta_{r} \stackrel{1 r}{\rightarrow} \delta_{r+1}$ & $Z_{1}^{(r)}$ & $Z_{2}^{(r)}$ & $Z_{5}^{(r)}$ & $Z_{6}^{(r)}$ \\
\hline$), 0,0,1) \stackrel{1 r}{\rightarrow}(0,0,0$ & $0,0,0, \nu) \stackrel{1 r}{\rightarrow}(\nu, 0, \nu, \nu)$ & - & . & - & $\{0,1\}$ \\
\hline 0$) \stackrel{1 r}{\rightarrow}(0$ & $(0,0, \nu, 0) \stackrel{1 r}{\rightarrow}(0,0, \nu, 0)$ & - & - & $\{0,1$ & $\{0,1\}$ \\
\hline 1) $\stackrel{1 r}{\rightarrow}(0$ & $(0,0, \nu, \nu) \stackrel{1 r}{\rightarrow}(\nu, 0,0, \nu)$ & - & - & $\{0,1\}$ & - \\
\hline$) \stackrel{1 r}{\rightarrow}(0$ & $(0, \nu, 0,0) \stackrel{1 r}{\rightarrow}(\nu, \nu, \nu, 0)$ & - & $\{0,1\}$ & - & $\{0,1\}$ \\
\hline 0,1$) \stackrel{1 r}{\rightarrow}(0,1$ & $(0, \nu, 0, \nu) \stackrel{1 r}{\rightarrow}(0, \nu, 0, \nu)$ & - & $\{0,1\}$ & - & - \\
\hline$) \stackrel{1 r}{\rightarrow}(0$ & $(0, \nu, \nu, 0) \stackrel{1 r}{\rightarrow}(\nu, \nu, 0,0)$ & - & $\{0,1\}$ & $\{0,1\}$ & - \\
\hline$) \stackrel{1 r}{\longrightarrow}(0$ & $(0, \nu, \nu, \nu) \stackrel{1 r}{\rightarrow}(0, \nu, \nu, \nu)$ & - & $\{0,1\}$ & $\{0,1$ & $\{0,1$ \\
\hline$\stackrel{1 r}{\rightarrow}(1$ & $(\nu, 0,0,0) \stackrel{1 r}{\rightarrow}(\nu, 0,0,0)$ & $\{0,1\}$ & - & $\{0,1$ & $\{0,1$ \\
\hline 1) $\stackrel{1 r}{\rightarrow}(1$ & $(\nu, 0,0, \nu) \stackrel{1 r}{\rightarrow}(0,0, \nu, \nu)$ & $\{0,1\}$ & - & $\{0,1$ & - \\
\hline 0$) \stackrel{1 r}{\rightarrow}(1$ & $\nu, 0) \stackrel{1 r}{\rightarrow}(\nu, 0, \nu, 0)$ & & - & - & - \\
\hline$\stackrel{1 r}{\longrightarrow}(1$ & $\nu, \nu) \stackrel{1 r}{\rightarrow}(0,0,0, \nu)$ & $\{0,1\}$ & - & - & $\{0,1\}$ \\
\hline $1 r$ & , 0$) \stackrel{1 r}{\rightarrow}(0, \nu, \nu, 0)$ & $\{0,1\}$ & $(0,1)$ & $\{0,1\}$ & - \\
\hline$\stackrel{1 r}{\rightarrow}(1$ & $(\nu, \nu, 0, \nu) \stackrel{1 r}{\rightarrow}(\nu, \nu, 0, \nu)$ & $\{0,1\}$ & (0) & $\{0,1$ & $\{0,1\}$ \\
\hline$\stackrel{1 r}{\rightarrow}(1,1,1,0)$ & $(\nu, \nu, \nu, 0) \stackrel{1 r}{\rightarrow}(0, \nu, 0,0)$ & $\{0,1\}$ & $\{0$. & - & $\{0,1\}$ \\
\hline$, 1,1,1) \stackrel{1 r}{\rightarrow}(1,1,1,1)$ & $(\nu, \nu, \nu, \nu) \stackrel{1 r}{\rightarrow}(\nu, \nu, \nu, \nu)$ & $\{0,1\}$ & & - & - \\
\hline
\end{tabular}

Table 3. The largest linear weak-key class of PES

\begin{tabular}{|c|c|c|c|}
\hline Round $r$ & Linear Relation & $\mathrm{msb}_{15}\left(Z_{6}^{(r)}\right)$ & Weak-Key Class Size \\
\hline 1 & $(0,0,0,1) \stackrel{1 r}{\rightarrow}(0,0,0,1)$ & $80-94$ & $2^{113}$ \\
2 & $(0,0,0,1) \stackrel{1 r}{\rightarrow}(0,0,0,1)$ & $73-87$ & $2^{106}$ \\
3 & $(0,0,0,1) \stackrel{1 r}{\rightarrow}(0,0,0,1)$ & $66-80$ & $2^{99}$ \\
4 & $(0,0,0,1) \stackrel{1 r}{\rightarrow}(0,0,0,1)$ & $34-48$ & $2^{84}$ \\
5 & $(0,0,0,1) \stackrel{1 r}{\rightarrow}(0,0,0,1)$ & $27-41$ & $2^{77}$ \\
6 & $(0,0,0,1) \stackrel{1 r}{\rightarrow}(0,0,0,1)$ & $20-34$ & $2^{70}$ \\
7 & $(0,0,0,1) \stackrel{1 r}{\rightarrow}(0,0,0,1)$ & $13-27$ & $2^{63}$ \\
8 & $(0,0,0,1) \stackrel{1 r}{\rightarrow}(0,0,0,1)$ & $109-123$ & $2^{48}$ \\
OT & $(0,0,0,1) \stackrel{0.5 r}{\rightarrow}(0,0,0,1)$ & - & $2^{48}$ \\
\hline
\end{tabular}

Appeared in Proceedings of the 6th Information Security Conference (ISC 2003), Lecture Notes in Computer Science 2851, C. Boyd, and W. Mao (eds.), Springer-Verlag, pp. 267-279, 2003. (C)2003 Springer-Verlag 
iterative characteristic $(\nu, 0, \nu, 0) \stackrel{1 r}{\rightarrow}(\nu, 0, \nu, 0)$ that holds with probability one, under some subkey restrictions (Table 4).

Table 4. Differential weak-key class of PES

\begin{tabular}{|c|c|c|c|}
\hline Round $r$ & Differential & $\mathrm{msb}_{15}\left(Z_{1}^{(r)}\right)$ & Weak-Key Class Size \\
\hline 1 & $(\nu, 0, \nu, 0) \stackrel{1 r}{\rightarrow}(\nu, 0, \nu, 0)$ & $0-14$ & $2^{113}$ \\
2 & $(\nu, 0, \nu, 0) \stackrel{1 r}{\rightarrow}(\nu, 0, \nu, 0)$ & $96-110$ & $2^{98}$ \\
3 & $(\nu, 0, \nu, 0) \stackrel{1 r}{\rightarrow}(\nu, 0, \nu, 0)$ & $89-103$ & $2^{91}$ \\
4 & $(\nu, 0, \nu, 0) \stackrel{1 r}{\rightarrow}(\nu, 0, \nu, 0)$ & $82-96$ & $2^{84}$ \\
5 & $(\nu, 0, \nu, 0) \stackrel{1 r}{\rightarrow}(\nu, 0, \nu, 0)$ & $75-89$ & $2^{77}$ \\
6 & $(\nu, 0, \nu, 0) \stackrel{1 r}{\rightarrow}(\nu, 0, \nu, 0)$ & $43-57$ & $2^{62}$ \\
7 & $(\nu, 0, \nu, 0) \stackrel{1 r}{\rightarrow}(\nu, 0, \nu, 0)$ & $36-50$ & $2^{55}$ \\
8 & $(\nu, 0, \nu, 0) \stackrel{1 r}{\rightarrow}(\nu, 0, \nu, 0)$ & $29-43$ & $2^{48}$ \\
OT & $(\nu, 0, \nu, 0) \stackrel{0.5 r}{\rightarrow}(\nu, 0, \nu, 0)$ & $22-36$ & $2^{41}$ \\
\hline
\end{tabular}

The differential weak-key class in Table 4 assumes that the user key bits numbered $0-14,22-57$ and $75-110$ are zero. The user key bits numbered 15-21, 58-74 and 111-127 can be arbitrary, which implies a differential weak-key class of size $2^{41}$. The characteristic in Table 4 can also be iterated beyond 8.5 rounds. For example, for 17 -round PES the one-round characteristic $(\nu, 0, \nu, 0) \stackrel{1 r}{\rightarrow}(\nu, 0, \nu, 0)$ holds for a weak-key class of size $2^{7}$. For 17.5 -round PES, this same characteristic holds only for the all-zero key, (the all-zero key is the only key for which this characteristic can be iterated for $r$-round PES for arbitrarily large $r$ ). Another differential weak-key class for PES is detailed in Table 5.

Table 5. Another differential weak-key class of PES

\begin{tabular}{|c|c|c|c|}
\hline Round $r$ & Differential & $\mathrm{msb}_{15}\left(Z_{2}^{(r)}\right)$ & Weak-Key Class Size \\
\hline 1 & $(0, \nu, 0, \nu) \stackrel{1 r}{\rightarrow}(0, \nu, 0, \nu)$ & $16-30$ & $2^{113}$ \\
2 & $(0, \nu, 0, \nu) \stackrel{1 r}{\rightarrow}(0, \nu, 0, \nu)$ & $112-126$ & $2^{98}$ \\
3 & $(0, \nu, 0, \nu) \stackrel{1 r}{\rightarrow}(0, \nu, 0, \nu)$ & $105-119$ & $2^{91}$ \\
4 & $(0, \nu, 0, \nu) \stackrel{1 r}{\rightarrow}(0, \nu, 0, \nu)$ & $98-112$ & $2^{84}$ \\
5 & $(0, \nu, 0, \nu) \stackrel{1 r}{\rightarrow}(0, \nu, 0, \nu)$ & $91-105$ & $2^{77}$ \\
6 & $(0, \nu, 0, \nu) \stackrel{1 r}{\rightarrow}(0, \nu, 0, \nu)$ & $59-73$ & $2^{62}$ \\
7 & $(0, \nu, 0, \nu) \stackrel{1 r}{\rightarrow}(0, \nu, 0, \nu)$ & $52-66$ & $2^{55}$ \\
8 & $(0, \nu, 0, \nu) \stackrel{1 r}{\rightarrow}(0, \nu, 0, \nu)$ & $45-59$ & $2^{48}$ \\
OT & $(0, \nu, 0, \nu) \stackrel{0.5 r}{\rightarrow}(0, \nu, 0, \nu)$ & $38-52$ & $2^{41}$ \\
\hline
\end{tabular}

Appeared in Proceedings of the 6th Information Security Conference (ISC 2003),

Lecture Notes in Computer Science 2851, C. Boyd, and W. Mao (eds.), Springer-Verlag, pp. 267-279, 2003. (C)2003 Springer-Verlag 
The differential weak-key class in Table 5 assumes that the user key bits numbered 16-30, 38-73, and 91-126 be zero. This leaves key bits 0-15, 31-37, $74-90$ and 127 to be arbitrary, and implies a weak-key class of size $2^{41}$, like in Table 4, but notice that both key classes are distinct, because they do not share the same restriction on several subkey bits.

\section{Differential-Linear Weak Keys}

In [4], Hawkes describes a differential-linear distinguisher for IDEA, under weakkey assumptions. His attack uses the characteristic depicted in Table 6, where $\delta_{1} \oplus \delta_{2}=\nu$. This characteristic holds with probability one, for a differentiallinear weak-key class of size $2^{63}$ for which key bits $19-28,72-124$ are arbitrary.

Table 6. Differential-linear relation of IDEA

\begin{tabular}{|c|c|c|c|c|}
\hline Round $r$ & Differential-Linear Relation & $\mathrm{msb}_{15}\left(Z_{1}^{(r)}\right)$ & $\mathrm{msb}_{15}\left(Z_{4}^{(r)}\right)$ & $\mathrm{msb}_{15}\left(Z_{5}^{(r)}\right)$ \\
\hline 1 & $(0, \nu, 0, \nu) \stackrel{1 r}{\rightarrow}(0,0, \nu, \nu)$ & - & $48-62$ & - \\
2 & $(0,0, \nu, \nu) \stackrel{1 r}{\rightarrow}(0, \nu, \nu, 0)$ & - & $41-55$ & $57-71$ \\
3 & $(0, \nu, \nu, 0) \stackrel{1 r}{\rightarrow}(0, \nu, 0, \nu)$ & - & - & $50-64$ \\
4 & $(0, \nu, 0, \nu) \stackrel{1 r}{\rightarrow}(0,0, \nu, \nu)$ & - & $2-16$ & - \\
5 & $(0,0, \nu, \nu) \stackrel{1 r}{\rightarrow}\left(\delta_{1}, \delta_{2}, \delta_{3}, \delta_{4}\right)$ & - & - & - \\
6 & $(1,1,0,0) \stackrel{1 r}{\rightarrow}(0,1,1,0)$ & $43-57$ & - & $4-18$ \\
7 & $(0,1,1,0) \stackrel{1 r}{\rightarrow}(1,0,1,0)$ & - & - & $125-11$ \\
8 & $(1,0,1,0) \stackrel{1 r}{\rightarrow}(1,1,0,0)$ & $29-43$ & - & - \\
\hline
\end{tabular}

For PES, one of the largest differential-linear distinguishers combines a 3round characteristic with a 5 -round linear relation. It is described in Table 7, where $\delta_{2} \oplus \delta_{4}=\nu$. This characteristic holds for a differential-linear weak-key class of size $2^{62}$, in which key bits $15-44,74-90$, and $113-127$ can be arbitrary.

A second differential-linear characteristic for PES, detailed in Table 8, holds for a weak-key class also of size $2^{62}$. Key bits numbered $15-44,74-88$, and $111-$ 127 can be arbitrary.

In [3] Daemen et al. suggested a modified key schedule for IDEA in order to avoid linear and differential attacks under weak-key assumptions. The modification consisted in xoring each subkey word with the constant value Odae $_{x}=0000110110101110_{2}$. This modified key schedule implies, under some weak-key assumptions, that some key bits be ' 0 ' and and under other assumptions that they be ' 1 '; a contradiction. This new key schedule avoids all differential, linear and differential-linear attacks shown previously for the 8.5-round PES and IDEA. For Daemen's redesigned key schedule the longest characteristic

Appeared in Proceedings of the 6th Information Security Conference (ISC 2003),

Lecture Notes in Computer Science 2851, C. Boyd, and W. Mao (eds.), Springer-Verlag, pp. 267-279, 2003. (C)2003 Springer-Verlag 
Table 7. Differential-linear relation of PES

\begin{tabular}{|c|c|c|c|}
\hline Round $r$ & Differential-Linear Relation & $\mathrm{msb}_{15}\left(Z_{1}^{(r)}\right)$ & $\mathrm{msb}_{15}\left(Z_{2}^{(r)}\right)$ \\
\hline 1 & $(\nu, 0, \nu, 0) \stackrel{1 r}{\rightarrow}(\nu, 0, \nu, 0)$ & $0-14$ & - \\
2 & $(\nu, 0, \nu, 0) \stackrel{1 r}{\rightarrow}(\nu, 0, \nu, 0)$ & $96-110$ & - \\
3 & $(\nu, 0, \nu, 0) \stackrel{1 r}{\rightarrow}\left(\delta_{1}, \delta_{2}, \delta_{3}, \delta_{4}\right)$ & - & - \\
4 & $(0,1,0,1) \stackrel{1 r}{\rightarrow}(0,1,0,1)$ & - & $98-112$ \\
5 & $(0,1,0,1) \stackrel{1 r}{\rightarrow}(0,1,0,1)$ & - & $91-105$ \\
6 & $(0,1,0,1) \stackrel{1 r}{\rightarrow}(0,1,0,1)$ & - & $59-73$ \\
7 & $(0,1,0,1) \stackrel{1 r}{\rightarrow}(0,1,0,1)$ & - & $52-66$ \\
8 & $(0,1,0,1) \stackrel{1 r}{\rightarrow}(0,1,0,1)$ & - & $45-59$ \\
\hline
\end{tabular}

Table 8. Another differential-linear relation of PES

\begin{tabular}{|c|c|c|c|}
\hline Round $r$ & Differential-Linear Relation & $\mathrm{msb}_{15}\left(Z_{1}^{(r)}\right)$ & $\mathrm{msb}_{15}\left(Z_{2}^{(r)}\right)$ \\
\hline 1 & $(\nu, 0, \nu, 0) \stackrel{1 r}{\rightarrow}(\nu, 0, \nu, 0)$ & $0-14$ & - \\
2 & $(\nu, 0, \nu, 0) \stackrel{1 r}{\rightarrow}(\nu, 0, \nu, 0)$ & $96-110$ & - \\
3 & $(\nu, 0, \nu, 0) \stackrel{1 r}{\rightarrow}(\nu, 0, \nu, 0)$ & $89-103$ & - \\
4 & $(\nu, 0, \nu, 0) \stackrel{1 r}{\rightarrow}\left(\delta_{1}, \delta_{2}, \delta_{3}, \delta_{4}\right)$ & - & - \\
5 & $(0,1,0,1) \stackrel{1 r}{\rightarrow}(0,1,0,1)$ & - & $91-105$ \\
6 & $(0,1,0,1) \stackrel{1 r}{\rightarrow}(0,1,0,1)$ & - & $59-73$ \\
7 & $(0,1,0,1) \stackrel{1 r}{\rightarrow}(0,1,0,1)$ & - & $52-66$ \\
8 & $(0,1,0,1) \stackrel{1 r}{\rightarrow}(0,1,0,1)$ & - & $45-59$ \\
\hline
\end{tabular}

Appeared in Proceedings of the 6th Information Security Conference (ISC 2003), Lecture Notes in Computer Science 2851, C. Boyd, and W. Mao (eds.), Springer-Verlag, pp. 267-279, 2003. (C)2003 Springer-Verlag 
identified for IDEA has 2.5 rounds, and is shown in Table $9 .^{1}$ It requires that the user key bits numbered 9-12, 15, 18, 20, 41-44, 47, 50, 52, 64-67, 70, 73, 75 be zero, and the user key bits numbered 13, 14, 16, 17, 19, 21-23, 45, 46, 48, 49, $51,53-55,68,69,71,72,74,76-78$ be one. In total, 45 key bits are restricted which represents a differential weak-key class of size $2^{128-45}=2^{83}$.

Table 9. 2.5-round differential for IDEA with Daemen's new key schedule

\begin{tabular}{|c|c|c|c|}
\hline Round $r$ & Differential & $\operatorname{msb}_{15}\left(Z_{1}^{(r)}\right)$ & $\operatorname{msb}_{15}\left(Z_{4}^{(r)}\right)$ \\
\hline 1 & $(0, \nu, \nu, 0) \stackrel{1 r}{\rightarrow}(0, \nu, 0, \nu)$ & $64-78$ & - \\
2 & $(0, \nu, 0, \nu) \stackrel{1 r}{\rightarrow}(0,0, \nu, \nu)$ & - & $41-55$ \\
2.5 & $(0,0, \nu, \nu) \stackrel{0.5 r}{\rightarrow}(0,0, \nu, \nu)$ & - & $9-23$ \\
\hline
\end{tabular}

The longest linear relation found for IDEA with the modified key schedule has 2.5 rounds. It requires that key bits in positions $2-5,8,11,13,34-37,40$, $43,45,66-69,72,75,77$ be zero, and key bits numbered $6,7,9,10,12,14-16$, $38,39,41,42,44,46-48,70,71,73,74,76,78-80$ be one. In total, 45 key bits are restricted which represent a class of $2^{83}$ weak keys (Table 10).

Table 10. 2.5-round linear relation for IDEA with Daemen's new key schedule

\begin{tabular}{|c|c|c|c|}
\hline Round $r$ & Linear Relation & $\mathrm{msb}_{15}\left(Z_{4}^{(r)}\right)$ & $\mathrm{msb}_{15}\left(Z_{6}^{(r)}\right)$ \\
\hline 3 & $(0,1,0,0) \stackrel{1 r}{\longrightarrow}(0,0,0,1)$ & - & $66-80$ \\
4 & $(0,0,0,1) \stackrel{1 r}{\longrightarrow}(0,0,1,0)$ & $2-16$ & $34-48$ \\
4.5 & $(0,0,1,0) \stackrel{0.5 r}{\rightarrow}(0,0,1,0)$ & - & - \\
\hline
\end{tabular}

The longest differential-linear relation found for IDEA with modified key schedule has 3.5 rounds and requires that key bits numbered $64-67,70,73,75$, $41-44,47,50,52,9-12,15,18,20,82-85,88,91,93$ be zero, and key bits 68,69 , $71,72,74,76-78,45,46,48,49,51,53-55,13,14,16,17,19,21-23,86,87,89,90$, $92,94-96$ be one. These restrictions on 60 key bits result in a differential-linear weak-key class of size $2^{68}$ (Table 11 ).

For PES with modified key schedule, the longest differential found has 2.5 rounds. It requires that key bits $66-69,7275,77,82-85,88,91,93,34-37,40$, 43, 45 be zero, and key bits 70, 71, 73, 74, 76, 78-80, 86, 87, 89, 90, 92, 94-96, $38,39,41,42,44,46-48$ be one. These 45 restricted bits represent a class of $2^{83}$ weak differential keys for PES (Table 12).

\footnotetext{
${ }^{1}$ We made a program to search exhaustively for the longest characteristic, and 2.5 rounds is the longest one found.
}

Appeared in Proceedings of the 6th Information Security Conference (ISC 2003),

Lecture Notes in Computer Science 2851, C. Boyd, and W. Mao (eds.), Springer-Verlag, pp. 267-279, 2003. (C)2003 Springer-Verlag 
Table 11. 3.5-round differential-linear relation for IDEA with Daemen's new key schedule

\begin{tabular}{|c|c|c|c|c|}
\hline Round $r$ & Differential or Lin. Relation & $\operatorname{msb}_{15}\left(Z_{1}^{(r)}\right)$ & $\operatorname{msb}_{15}\left(Z_{4}^{(r)}\right)$ & $\operatorname{msb}_{15}\left(Z_{5}^{(r)}\right)$ \\
\hline 1 & $(0, \nu, \nu, 0) \stackrel{1 r}{\rightarrow}(0, \nu, 0, \nu)$ & - & - & $64-78$ \\
2 & $(0, \nu, 0, \nu) \stackrel{1 r}{\rightarrow}(0,0, \nu, \nu)$ & - & $41-55$ & - \\
3 & $(0,0, \nu, \nu) \stackrel{1 r}{\longrightarrow}\left(\delta_{1}, \delta_{2}, \delta_{3}, \delta_{4}\right)$ & - & $9-23$ & - \\
3.5 & $(1,0,1,0) \stackrel{0.5 r}{\longrightarrow}(1,1,0,0)$ & - & - & - \\
4 & $(1,1,0,0) \stackrel{1 r}{\rightarrow}(1,1,0,0)$ & $82-96$ & - & - \\
\hline
\end{tabular}

Table 12. 2.5-round differential for PES with Daemen's new key schedule

\begin{tabular}{|c|c|c|c|}
\hline Round $r$ & Differential & $\mathrm{msb}_{15}\left(Z_{1}^{(r)}\right)$ & $\mathrm{msb}_{15}\left(Z_{6}^{(r)}\right)$ \\
\hline 3 & $(0,0,0, \nu) \stackrel{1 r}{\rightarrow}(\nu, 0, \nu, \nu)$ & - & $66-80$ \\
4 & $(\nu, 0, \nu, \nu) \stackrel{1 r}{\rightarrow}(0,0,0, \nu)$ & $82-96$ & $34-48$ \\
4.5 & $(0,0,0, \nu) \stackrel{0.5 r}{\rightarrow}(0,0,0, \nu)$ & - & - \\
\hline
\end{tabular}

The longest linear relation found for PES with Daemen's new key schedule has 2.5 rounds. It requires that key bits in positions $66-69,72,75,77,34-37,40$, 43, 45 be zero, and key bits numbered 70, 71, 73, 74, 76, 78-80, 38, 39, 41, 42, $44,46-48$ be one. These restrictions on 30 key bits represent a weak-key class of size $2^{98}$ (Table 13 ).

Table 13. 2.5-round linear relation for PES with Daemen's new key schedule

\begin{tabular}{|c|c|c|}
\hline Round $r$ & Linear Relation & $\mathrm{msb}_{15}\left(Z_{6}^{(r)}\right)$ \\
\hline 3 & $(0,0,0,1) \stackrel{1 r}{\rightarrow}(0,0,0,1)$ & $66-80$ \\
4 & $(0,0,0,1) \stackrel{1 r}{\longrightarrow}(0,0,0,1)$ & $34-48$ \\
4.5 & $(0,0,0,1) \stackrel{0.5 r}{\rightarrow}(0,0,0,1)$ & - \\
\hline
\end{tabular}

\section{Conclusions}

One linear weak-key class of size $2^{48}$, and two differential weak-key classes, both of size $2^{41}$, were identified for 8.5-round PES, in a similar setting as done by Daemen et al. for the IDEA cipher [3]. It was observed that linear and differential weak-keys can be identified for up to 17 rounds of PES, and up to 14 rounds of IDEA. These attacks on extended-round variants of PES and IDEA demonstrate that doubling the number of rounds is not enough to avoid weak keys. This is a consequence of the key overlapping property in the key schedule algorithm. A redesign of the latter is recommended, in order to avoid the simple mapping between subkeys and the user key.

Appeared in Proceedings of the 6th Information Security Conference (ISC 2003),

Lecture Notes in Computer Science 2851, C. Boyd, and W. Mao (eds.), Springer-Verlag, pp. 267-279, 2003. (C)2003 Springer-Verlag 
For IDEA, Daemen et al. described a linear weak-key class of size $2^{23}$, and a differential weak-key class of size $2^{51}$.

Two differential-linear weak-key classes for PES were identified, each with size $2^{62}$. The largest differential-linear characteristic described by Hawkes in [4] has size $2^{63}$.

Finally, it was also found that linear weak keys of size $2^{10}$ exist for 17 -round PES, which is equivalent to double PES. Moreover, a differential weak-key class of size $2^{7}$ was identified for 17 -round PES. Table 14 summarizes the results of this paper. Notice that even though the key schedule is the same, the weak-key classes are different for PES and IDEA, both in size and in the key bit patterns. This is a consequence of the round structure of these ciphers, Fig.s 1 and 2, and the fact that the weak-key restrictions are derived only from the multiplicative subkeys (in the differential case, the difference word $\nu=8000_{x}$ propagates with probability one across the subkey addition for any additive subkey).

The new key schedule of IDEA and PES suggested by Daemen is quite effective against differential, linear, differential-linear and also against boomerang attacks [2], all of which exploit the same key overlapping property of the key schedule. Our search program could find only a 2.5 -round differential and linear relation for them with the modified key setup algorithm. Moreover, a 3.5-round differential-linear relation for IDEA was also discovered. These reduced-round differential, linear and differential-linear attacks did not necessarily start from the first cipher round, but in some intermediate round along the cipher in order to explore the largest overlap of user key bits. Therefore, both the original and modified key schedules of PES and IDEA imply that different rounds of these ciphers do not present the same level of security. This fact was already observed by Biham et al. in Impossible Differential attacks [1]. Distinct rounds imply different constraints on the user key bits, resulting in larger or smaller weak-key classes. The larger the weak-key class the better the attack because it means the attack applies to more keys.

Implications of weak keys in block ciphers such as PES and IDEA include:

- the cipher(s) can be distinguished from a random permutation, with a small computational effort, although the attacks apply only to some particular weak-key classes;

- we emphasize that all weak keys are derived from the original key schedule algorithms of PES and IDEA. Therefore, these are all legal user keys, without any tweak on the key setup procedure;

- attacks using weak keys can reach the full 8.5-round (and even longer versions of) PES and IDEA. Without weak-key assumptions there are no known attacks reported in the literature that can reach more than 4.5 rounds of IDEA [1];

- for reduced-round cipher versions, such as 3.5-round IDEA with Daemen's new key schedule, a key-recovery attack can be performed on larger cipher versions (e.g. 4-round IDEA), in order to discover unknown user key bits;

- in stream cipher constructions using block ciphers, such as in OFB mode of operation [9], weak keys can generate streams of very small period. Consider

Appeared in Proceedings of the 6th Information Security Conference (ISC 2003),

Lecture Notes in Computer Science 2851, C. Boyd, and W. Mao (eds.), Springer-Verlag, pp. 267-279, 2003. (C)2003 Springer-Verlag 
the all-zero user key. In IDEA and PES this weak user key implies that the encryption and decryption processes are the same (because the encryption and decryption subkeys are all equal). Let the encryption operation be denoted $E_{k}$, where $E_{k} \in\left\{\mathrm{IDEA}_{k}, \mathrm{PES}_{k}\right\}$. Therefore, for any initial value (IV) to the OFB mode, the output stream will have a cycle of length two with values $E_{0}(\mathrm{IV})$, and $E_{0}\left(E_{0}(\mathrm{IV})\right)=\mathrm{IV}$;

- the linear weak-key class of IDEA found by Daemen et al. has size $2^{23}$, and the differential weak-key class has size $2^{51}$. It means that if the user keys are chosen uniformly at random, there is a chance of $2^{-105}$ (resp. $2^{-77}$ ) that such weak keys would ever be used. Moreover, it can be argued that the simple mapping from subkeys to user keys allows to easily identify all weak user keys according to some bit pattern, and therefore, such user keys can be immediately discarded in case they are generated. But in hash function constructions based on block ciphers, such as in Davies-Meyer [9], the message blocks are input as key values. Let the message blocks be denoted $x_{i}$, and the intermediate hash values be denoted $H_{i}, 0 \leq i \leq n$. Let $E_{k}=\mathrm{IDEA}_{k}$. Then, in Davies-Meyer construction, $H_{0}=$ IV (initial value), and

$$
H_{i}=H_{i-1} \oplus E_{x_{i-1}}\left(H_{i-1}\right) .
$$

It can be verified that $E_{0}(1,0,1,1)=(0,1,0,0)$, where the 4-tuples represent 64-bit blocks. Furthermore, $E_{0}(0,1,0,0)=E_{0}\left(E_{0}(1,0,1,1)\right)=(1,0,1,1)$. Therefore, if one sets $x_{0}=0$, then $H_{0}=(1,0,1,1)$ and $H_{0}^{\prime}=(0,1,0,0)$ will both result in $H_{1}=(1,1,1,1)$, which is an internal collision. Morever, the differential, linear and differential-linear weak-key classes for PES and IDEA are distinct, and there are several of them. The ones described in this report are only the largest ones found. It means that, if weak user keys are to be discarded based on some bit pattern, there are several weak-key classes to be tested, which may slow down subkey generation, and may provide an opportunity for some kind of timing attack [5].

\section{Acknowledgements}

We would like to thank the anonymous referees for the many suggestions that helped improve the presentation of this paper.

\section{References}

1. Biham, E., Biryukov, A., Shamir, A., Miss-in-the-Middle Attacks on IDEA, Khufu and Khafre, In: Knudsen, L.R. (ed.): 6th Fast Software Encryption Workshop, Lecture Notes in Computer Science, Vol. 1636. Springer-Verlag, Berlin Heidelberg New York (1999), 124-138.

2. Biryukov, A., Nakahara Jr, J., Preneel, B., Vandewalle, J.: New Weak-Key Classes of IDEA, In: Deng,R., Qing, S., Bao,F., Zhou,J. (eds): International Conference on Information and Communications Security, ICICS 2002, Singapore, Lecture Notes in Computer Science, Vol. 2513. Springer-Verlag, Berlin Heidelberg New York (2002), 315-326.

Appeared in Proceedings of the 6th Information Security Conference (ISC 2003),

Lecture Notes in Computer Science 2851, C. Boyd, and W. Mao (eds.), Springer-Verlag, pp. 267-279, 2003. (C)2003 Springer-Verlag 
Table 14. Summary of attacks on IDEA, PES and extended variants

\begin{tabular}{|c|c|c|}
\hline Cipher & Weak-Key Class Size & Type of Attack \\
\hline \hline 8.5-round PES & $2^{48}$ & Linear \\
& $2^{41}$ & Differential \\
& $2^{62}$ & Diff.-Linear \\
\hline 16-round PES & $2^{10}$ & Linear \\
\hline 17-round PES & $2^{7}$ & Linear \\
& $2^{7}$ & Differential \\
\hline 14-round IDEA & $2^{4}$ & Linear \\
\hline 2.5-round Daemen's IDEA & $2^{83}$ & Differential \\
& $2^{83}$ & Linear \\
\hline 3.5-round Daemen's IDEA & $2^{68}$ & Diff.-Linear \\
\hline 2.5-round Daemen's PES & $2^{83}$ & Differential \\
& $2^{98}$ & Linear \\
\hline
\end{tabular}

3. Daemen, J., Govaerts, R., Vandewalle, J.: Weak Keys for IDEA, In: Stinson, D.R. (ed.): Advances in Cryptology, Proceedings of Crypto 93, Lecture Notes in Computer Science, Vol. 773. Springer-Verlag, Berlin Heidelberg New York (1994), 224231.

4. Hawkes, P.M.: Differential-Linear Weak Key Classes of IDEA, In: Nyberg, K. (ed.): Advances in Cryptology, Proceedings of Eurocrypt 98, Lecture Notes in Computer Science, Vol. 1403. Springer-Verlag, Berlin Heidelberg New York (1998), 112-126.

5. Kocher, P.C., Timing Attacks on Implementations of Diffie-Hellman, RSA, DSS, and Other Systems, In: Koblitz, N. (ed): Advances in Cryptology, Crypto'96, Lecture Notes in Computer Science, Vol. 1109, Springer-Verlag, Berlin Heidelberg New York (1996), 104-113

6. Lai, X., Massey, J.L.: A Proposal for a New Block Encryption Standard, in: Damgård, I.B. (ed.): Advances in Cryptology, Proceedings of Eurocrypt 90, Lecture Notes in Computer Science, Vol. 473. Springer-Verlag, Berlin Heidelberg New York (1990), 389-404.

7. Lai, X., Massey, J.L., Murphy, S.: Markov Ciphers and Differential Cryptanalysis, In: Davies,D.W. (ed.): Advances in Cryptology, Eurocrypt 91, Lecture Notes in Computer Science, Vol. 547. Springer-Verlag, Berlin Heidelberg New York (1991), $17-38$.

8. Mediacrypt AG: The IDEA Block Cipher, Submission to the NESSIE Project at http://cryptonessie.org.

9. Menezes, A.J., van Oorschot, P.C., Vanstone, S., Handbook of Applied Cryptography, CRC Press, 1997.

10. NESSIE Project: New European Schemes for Signatures, Integrity and Encryption at http://cryptonessie.org.

Appeared in Proceedings of the 6th Information Security Conference (ISC 2003),

Lecture Notes in Computer Science 2851, C. Boyd, and W. Mao (eds.), Springer-Verlag, pp. 267-279, 2003. (C)2003 Springer-Verlag 